\title{
Model for nucleation in GaAs homoepitaxy derived from first principles
}

\author{
P. Kratzer \\ Fritz-Haber-Institut der Max-Planck-Gesellschaft, Faradayweg 4-6, D-14195 Berlin, Germany \\ C. G. Morgan \\ Fritz-Haber-Institut der Max-Planck-Gesellschaft, Faradayweg 4-6, D-14195 Berlin, Germany \\ and Physics Department, Wayne State University, Detroit, Michigan 48202 \\ M. Scheffler \\ Fritz-Haber-Institut der Max-Planck-Gesellschaft, Faradayweg 4-6, D-14195 Berlin, Germany
}

(Received 15 December 1998)

\begin{abstract}
The initial steps of molecular beam epitaxy growth of GaAs on $\beta 2$-reconstructed $\mathrm{GaAs}(001)$ are investigated by performing total energy and electronic structure calculations using density functional theory and a repeated slab model of the surface. We study the interaction and clustering of adsorbed $\mathrm{Ga}$ atoms and the adsorption of $\mathrm{As}_{2}$ molecules onto $\mathrm{Ga}$ atom clusters adsorbed on the surface. The stable nuclei consist of bound pairs of $\mathrm{Ga}$ adatoms, which originate either from dimerization or from an indirect interaction mediated through the substrate reconstruction. $\mathrm{As}_{2}$ adsorption is found to be strongly exothermic on sites with a square array of four $\mathrm{Ga}$ dangling bonds. Comparing two scenarios where the first $\mathrm{As}_{2}$ gets incorporated in the incomplete surface layer, or alternatively in a new added layer, we find the first scenario to be preferable. In summary, the calculations suggest that nucleation of a new atomic layer is most likely on top of those surface regions where a partial filling of trenches in the surface has occurred before. [S0163-1829(99)07719-X]
\end{abstract}

\section{INTRODUCTION}

Growth of well-ordered crystals of III-V compound semiconductors requires the incorporation of both constituents in the correct stoichiometric amounts. For instance, in molecular beam epitaxy (MBE) growth of GaAs, the Ga atoms and As molecules provided by the beam sources must be adsorbed and incorporated into the growing surface in such a way that the surface stoichiometry is locally maintained. The atomistic processes behind stoichiometric growth are complex and not yet fully understood at present. Moreover, the substrate surfaces used for growth of arsenide compound semiconductors, in particular the frequently used GaAs(001) surface, show a variety of complex surface reconstructions. Under moderately arsenic-rich conditions, as are commonly used during growth, the $\mathrm{GaAs}(001)$ surface displays reconstructions with a $(2 \times 4)$ symmetry: the $\alpha, \beta$, and $\beta 2$ reconstructions, which contain surface As dimers as common building blocks. The strongly corrugated $\beta 2$ reconstruction, which exposes three layers of atoms, prevails in a wide range of growth conditions and serves as the starting configuration for growth on the $\mathrm{GaAs}(001)$ substrate. For a well-controlled growth, it is required that this structure recovers after film deposition, at least after a short growth interruption. However, it was already understood in early growth models ${ }^{1}$ that different reconstructions may appear locally on the growing surface, acting as metastable intermediates before a newly grown layer is completed. The details of these structural transformations remained unclear until recently. Only with the help of detailed scanning tunnel microscope (STM) studies $^{2}$ has it become possible to refine our understanding of the elementary steps of growth. ${ }^{3}$ STM pictures taken from samples after submonolayer deposition followed by a fast quench to room temperature show two major processes contributing to growth on the $\beta 2$-reconstructed surface: the filling of trenches and the formation of small islands that later become part of the top-layer As dimers in the new layer. In the present paper, we present results of $a b$ initio calculations for the atomistic steps of these two growth scenarios.

\section{CAlCulations}

We performed $a b$ initio calculations using density functional theory to describe exchange and correlation in the electronic many-particle system. A recent version ${ }^{4}$ of the generalized-gradient approximation for the exchangecorrelation functional was employed. All calculations were done with slabs consisting of seven or eight atomic layers and a $4 \times 4$ lateral unit cell. The bottom layer was passivated with pseudohydrogen atoms and kept fixed, while the top six or seven layers and adatoms were allowed to relax.

Our calculations use fully separable, norm-conserving pseudopotentials ${ }^{5-7}$ to describe the electron-ion interaction, constructed from an all-electron atomic calculation with the generalized-gradient approximation (GGA) functional. ${ }^{4}$ Gonze's analysis ${ }^{8}$ was used to confirm that unphysical ghost states were not present in the separable representation. The wave functions were expanded in a plane wave basis with a cutoff energy of $10 \mathrm{Ry}$, and the $k$-space integration was performed with a special $\mathbf{k}$-point set, with a density equivalent to $64 \mathbf{k}$ points in the Brillouin zone of the $(1 \times 1)$ surface unit cell.

\section{ADSORPTION}

Both in thermodynamic equilibrium and over a wide range of growth conditions, the $\mathrm{GaAs}(001)$ surface forms the 

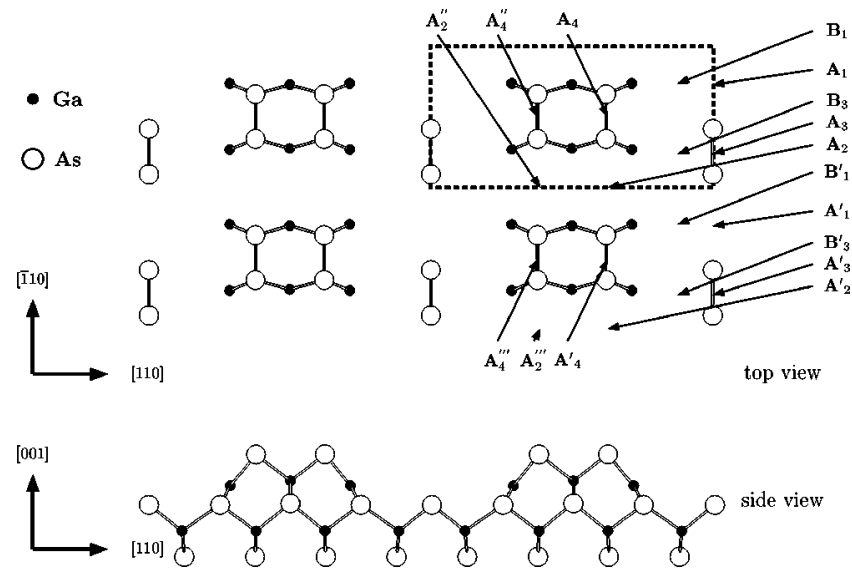

FIG. 1. Adsorption sites for $\mathrm{Ga}$ atoms on the $\beta 2(2 \times 4)$ reconstructed $\mathrm{GaAs}(001)$ surface. The As and Ga atoms of the substrate are displayed as white and black circles. The unit cell of the reconstruction is marked by the dashed rectangle. Adsorption sites are labeled within the $4 \times 4$ cell used in the calculations.

$\beta 2(2 \times 4)$ reconstruction. Each $(2 \times 4)$ unit cell is built up from two As dimers and two missing dimers in the topmost layer and a missing Ga pair in the second layer (see Fig. 1).

The missing atoms give rise to trenches running in the [ $\overline{1} 10]$ direction separated by mountains of adjacent As dimers in the top layer.

The As atoms in the third layer exposed in the trenches also form dimers. On the atomic level, one can imagine two principal ways in which growth can proceed on this surface. One possibility is the nucleation of new layers on top of the existing "mountains." Alternatively, the trenches could be filled up first, either partially or completely, before nucleation of new layers starts afterwards in these surface regions.

The adsorption of single gallium atoms on the $\mathrm{GaAs}(001)$ surface has been studied previously by means of density functional theory calculations. ${ }^{9}$ On the $\beta 2$-reconstructed surface, a $\mathrm{Ga}$ atom preferentially adsorbs between two As dimers in line with the dimer axis, at adsorption sites $A_{1}$ in the trench or in $A_{2}$ in the top layer, see Fig. 1 and Fig. 2(a). In these calculations, the substrate atoms were allowed to relax after deposition of the $\mathrm{Ga}$ adatom, but the bonding topology of the substrate atoms was maintained. A different kind of adsorption site arises when breaking of substrate

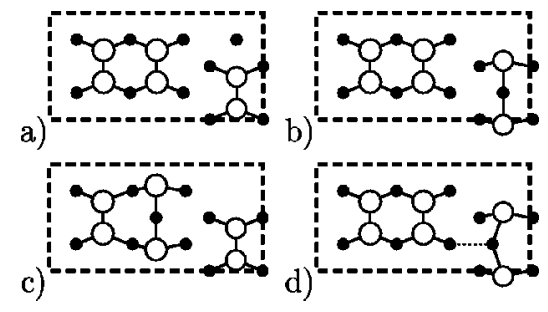

FIG. 2. Schematic illustration of some adsorption sites of Ga and accompanying substrate rearrangements on the $\mathrm{GaAs}(001) \beta 2$ surface: (a) Ga adatom in site $A_{1}$ between As dimers in the trench, (b) twofold coordinated Ga adatom in site $A_{3}$ splitting an As trench dimer, (c) twofold coordinated Ga adatom in site $A_{4}$ splitting an As dimer in the top layer, (d) threefold coordinated $\mathrm{Ga}$ adatom in site $B_{3}$ in the trench with additional bond to one side wall (dashed). As atoms are shown as white circles, Ga atoms as black circles.
TABLE I. Interaction energy of clusters of Ga adatoms in the trenches (left) and in the top layer (right) of the $\beta 2$ reconstruction. All numbers are given relative to isolated Ga adatoms in $A_{3}$ sites, with negative numbers indicating attractive interaction, with accuracy $50 \mathrm{meV}$.

\begin{tabular}{lrrr}
\hline \hline \multicolumn{2}{c}{ Trench } & \multicolumn{2}{c}{ Top layer } \\
\hline Singles & $\Delta E(\mathrm{eV})$ & & $\Delta E(\mathrm{eV})$ \\
\hline$A_{1}$ & 0.55 & $A_{2}$ & 0.60 \\
$B_{1}$ & 0.20 & $A_{4}$ & 0.20 \\
$B_{3}$ & 0.15 & & \\
& & & \\
Pairs & & & 0.20 \\
\hline$A_{3} A_{3}^{\prime}$ & 0.05 & $A_{4} A_{4}^{\prime}$ & 0.20 \\
$A_{3} A_{1}$ & 0.90 & $A_{4} A_{2}$ & -1.15 \\
$B_{3} B_{3}^{\prime}$ & 0.25 & $A_{4} A_{4}^{\prime \prime}$ & \\
$B_{3} B_{1}$ & -0.65 & &
\end{tabular}

Triples

\begin{tabular}{lrlr}
\hline$A_{3} A_{1} A_{3}^{\prime}$ & 0.00 & $A_{4} A_{2} A_{4}^{\prime}$ & 0.10 \\
$B_{3} B_{1} B_{3}^{\prime}$ & -1.00 & $A_{4} A_{2} A_{4}^{\prime \prime}$ & -0.65 \\
& & & \\
Quadruples & & & -2.20 \\
\hline & & $A_{4} A_{4}^{\prime \prime} A_{4}^{\prime} A_{4}^{\prime \prime \prime}$ & -0.35 \\
\hline
\end{tabular}

bonds is taken into consideration. The Ga atom may split an As dimer in the trench $\left[A_{3}\right.$, see Fig. 2(b)] or in the top layer $\left[A_{4}\right.$, see Fig. 2(c) $]$ and adsorb in a twofold coordinated site. ${ }^{9}$ $\mathrm{Ga}$ atoms in these sites are much more strongly bound than in the $A_{1}$ and $A_{2}$ sites. Adsorption there already constitutes the first step to incorporation of the Ga atoms. Moreover, the binding energy is higher in site $A_{3}$ in the trench than in the $A_{4}$ mountain site. As an alternative to adsorption sites $A_{1}$ and $A_{3}$, the $\mathrm{Ga}$ atom bonding to the As atoms in the trench may tilt away from the As dimer axis and form an additional bond with a $\mathrm{Ga}$ atom at the side wall of the trench $\left[B_{1}\right.$ and $B_{3}$, see Fig. 2(d)]. In this way the Ga atom reaches a threefold coordination. Our calculations with the GGA functional show that single threefold sites are less strongly bound than the corresponding twofold site $A_{3}$ (see Table I).

\section{NUCLEATION}

MBE growth of GaAs is usually performed by applying a flux of arsenic molecules that exceeds the flux of $\mathrm{Ga}$ atoms. Under these conditions the growth rate is mostly controlled by the diffusion and incorporation of the Ga atoms, while As is easily available everywhere on the surface. Therefore, we focus on the interaction of $\mathrm{Ga}$ adatoms on the surface that is mainly responsible for nucleation.

Gallium adsorption at site $A_{3}$ has the largest binding energy. Therefore, in the limit of low Ga coverage and low mobility, most of the deposited Ga atoms will get incorporated at randomly distributed $A_{3}$ sites. At growth temperatures, however, thermally activated jumps of Ga adatoms from $A_{3}$ into neighboring sites occur frequently, with a rate 


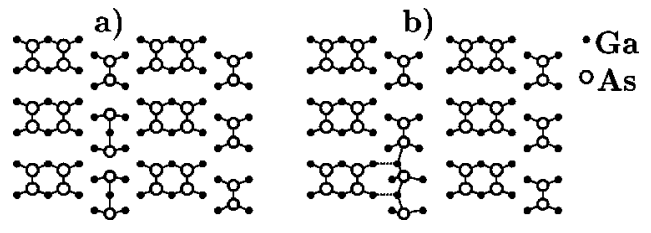

FIG. 3. "Trench" adsorption geometries (cf. left column in Table I) for Ga atoms in a trench: (a) two Ga atoms in sites $A_{3} A_{3}^{\prime}$ splitting two As dimers, (b) in adjacent $B_{3} B_{1}$ sites, thereby splitting only one As dimer.

of $\sim 10^{5} \mathrm{~s}^{-1} \cdot{ }^{10}$ In this way, other adsorption sites, though being higher in energy (see Table I), will become populated according to a thermal distribution. If Ga adatoms in neighboring adsorption sites interact attractively, clusters of $\mathrm{Ga}$ adatoms will form which act as precursors of island growth. Without substantial attraction, entropic effects lead to a preference for isolated single adsorbed $\mathrm{Ga}$ atoms in the small coverage limit.

We have studied the interaction between Ga adatoms for various configurations. The interaction energies are defined with respect to the adsorption of individual Ga atoms in isolated $A_{3}$ sites:

$$
\Delta E\left(A_{1}, \ldots A_{N}\right)=E\left(A_{1}, \ldots A_{N}\right)-N E\left(A_{3}\right) .
$$

The left column in Table I shows the interaction energy for $\mathrm{Ga}$ atoms adsorbed in the trench. The Ga atoms alternatingly occupy adjacent sites of the type $A_{1}$ and $A_{3}$, or $B_{1}$ and $B_{3}$. In this way, $\mathrm{Ga}-\mathrm{As}-\mathrm{Ga} \ldots$ chains in [110] direction are formed from the As dimers in the trenches. From the calculated energetics, we derive the following growth scenario: When a diffusing $\mathrm{Ga}$ adatom approaches another $\mathrm{Ga}$ adatom in an $A_{3}$ site, they can stabilize each other by forming a $B_{3} B_{1}$ pair. This process results in a local $\alpha$ reconstruction in one particular $(2 \times 4)$ unit cell. Although the Ga atoms do not form bonds with each other, such a pair is bound by $0.65 \mathrm{eV}$. Attachment of a third $\mathrm{Ga}$ adatom increases the interaction energy by only $0.35 \mathrm{eV}$ to $1.0 \mathrm{eV}$. The unexpected stability of a Ga pair is due to the fact that an As dimer bond is restored when the diffusing $\mathrm{Ga}$ atom moves from the next-nearest-neighbor site to the nearest-neighbor site with respect to a $\mathrm{Ga}$ atom adsorbed in the $A_{3}$ site (see Fig. 3 for illustration).

Since the clustering of only two Ga atoms already results in a large increase in the binding energy, we expect the rate of formation of such pairs from a lattice gas of single diffusing $\mathrm{Ga}$ adatoms to exceed the rate of break-up of pairs even for a moderate supersaturation of $\mathrm{Ga}$ on the surface. Thus our calculations demonstrate that the Ga adatom pair in the trench acts as a stable nucleus in the sense of nucleation theory.

Next we consider the alternative scenario, nucleation of a new layer without previous trench filling. The calculated results are collected in the right column of Table I. In general, $\mathrm{Ga}$ adsorption in the top-layer As dimers is energetically less favorable than adsorption in the trench dimers. Therefore, population of the top-layer sites is considerably smaller for low coverages, and only increases when the trench dimer sites are mostly occupied. However, when two Ga atoms adsorb in neighboring parallel As dimers, they reach a stable configuration due to the formation of a Ga dimer $\left(A_{4} A_{4}^{\prime \prime}\right.$ in Table I). The decay of the Ga dimer into two single $\mathrm{Ga}$ adatoms in $A_{3}$ sites is an endothermic process. This relative stability is maintained for those larger clusters that allow for formation of $\mathrm{Ga}$ dimers oriented perpendicularly to the previous As dimers in the layer below, i.e., the $A_{4} A_{2} A_{4}^{\prime \prime}$ and $A_{4} A_{4}^{\prime \prime} A_{4}^{\prime} A_{4}^{\prime \prime \prime}$ clusters. We note that the latter cluster has exactly twice the binding energy of a single Ga dimer, $A_{4} A_{4}^{\prime \prime}$. Thus, there is no extra attractive interaction between Ga dimers. If larger islands of $\mathrm{Ga}$ ad-dimers form on surface regions where the trenches are already filled up with Ga atoms, this is not due to an attractive interaction. However, such $\mathrm{Ga}$ islands may form accidentally and survive due to a limited mobility of the dimerized $\mathrm{Ga}$ atoms constituting these clusters.

After a cluster of Ga adatoms has formed in the trench, any further $\mathrm{Ga}$ adatoms that are deposited on the adjacent mountains will be more likely to remain on the mountains long enough to form Ga dimers there, since they would have to move further to find empty trench sites to occupy. This suggests that the formation of a Ga cluster in the trench acts to promote the subsequent formation of Ga dimer pairs on the adjacent mountains.

In contrast to the situation in the trench, the formation of $\mathrm{Ga}-\mathrm{As}-\mathrm{Ga} \ldots$ chains in the [110] direction by splitting top-layer As dimers is associated with only a minor gain in binding energy (clusters $A_{4} A_{2}, A_{4} A_{2} A_{4}^{\prime}$ ). Relative to individual $\mathrm{Ga}$ atoms sitting in top-layer As dimers ( $A_{4}$ sites), forming a chain containing two (three) $\mathrm{Ga}$ atoms gives a binding energy of $0.2 \mathrm{eV}(0.5 \mathrm{eV})$. However, these structures are unstable against decay into individual Ga adatoms in $A_{3}$ trench sites, and therefore should have little relevance for growth.

\section{THE ROLE OF ARSENIC}

Up to now, we have not considered the possibility of enhanced stability of the above structures due to adsorption of arsenic. As sources of arsenic in MBE growth, both $\mathrm{As}_{2}$ and $\mathrm{As}_{4}$ molecular beams are in use. For the issue of enhanced stability due to arsenic, it is sufficient to consider the simpler case of $\mathrm{As}_{2}$ adsorption. While $\mathrm{As}_{4}$ is believed to split into fragments upon adsorption, the $\mathrm{As}_{2}$ molecule can become incorporated into the $\beta 2(2 \times 4)$ structure without dissociation. $A b$ initio calculations ${ }^{11,12}$ show that the binding energy of chemisorbed $\mathrm{As}_{2}$ depends very much on the local environment. On an ideal $\beta 2$-reconstructed surface, an arsenic ad dimer is bound to the top-layer As dimers by $1.65 \mathrm{eV} .{ }^{11} \mathrm{It}$ can be shown that As ad-dimers bound to Ga atoms will stay permanently adsorbed even at standard growth temperatures, while As dimers that bind to As atoms or to only one Ga atom are more weakly bound and will either desorb or react with diffusing Ga atoms. ${ }^{11}$ This is consistent with the experimental observation that arsenic incorporation at these temperatures only proceeds when the surface is simultaneously exposed to a $\mathrm{Ga}$ beam providing excess $\mathrm{Ga}$ adatoms on the surface. ${ }^{13}$

We expect that enhanced stability due to $\mathrm{As}_{2}$ adsorption is most relevant for those structures containing L-shaped patches of three $\mathrm{Ga}$ atoms or rectangular patches of four $\mathrm{Ga}$ 
a)

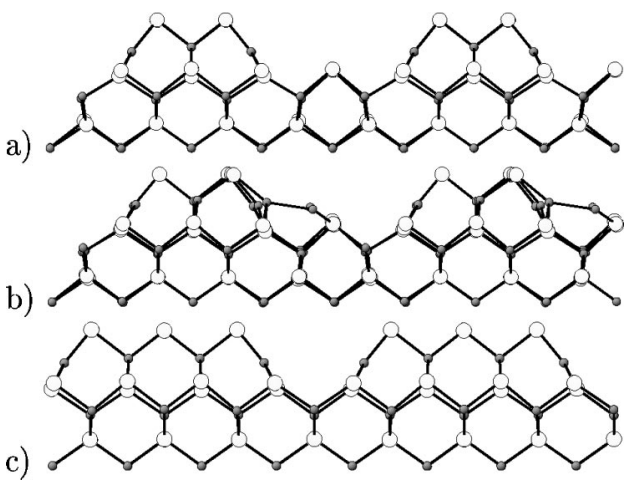

FIG. 4. Growth scenario for filling of the trenches on the clean $\beta 2$ surface (a), via formation of Ga atom pairs $B_{3} B_{1}$ (b), followed by $\mathrm{As}_{2}$ adsorption that leads to a local $\beta$ reconstruction (c). The pictures presented show part of the slab in side view, with relaxed atomic geometries as obtained from the calculations.

atoms in adjacent sites. In these local environments, an adsorbing $\mathrm{As}_{2}$ molecule can attach to the surface by building up three or four As-Ga backbonds. For the nucleation scenario in the trenches, such a situation already occurs for a single $\mathrm{Ga}$ atom adsorbed in the trench, for instance in the $A_{3}$ site. The $\mathrm{As}_{2}$ forms one backbond to this $\mathrm{Ga}$ atom, while backbonds with two other dangling orbitals of $\mathrm{Ga}$ atoms at the sidewall of the trench are simultaneously established. The binding energy of an $\mathrm{As}_{2}$ molecule relative to the gas phase is $1.9 \mathrm{eV}$ at this site, too low to make it permanently adsorbed at frequently used growth temperatures. When two $\mathrm{Ga}$ atoms are on adjacent sites in the trench, like in the local $\alpha$ structure [cluster $B_{3} B_{1}$, see also Fig. 4(b)], the adsorbing $\mathrm{As}_{2}$ can establish four backbonds and will transform this structure to the $\beta$ reconstruction, which contains three parallel As dimers [see Fig. 4(c)]. The binding energy of $\mathrm{As}_{2}$ is $2.4 \mathrm{eV}$ in this environment. ${ }^{11}$

Further $\mathrm{As}_{2}$ adsorption on the local $\beta$ reconstruction, which would lead to a complete filling of the trench, has been found to be energetically unfavorable. We find a binding energy of only $0.9 \mathrm{eV}$ for an As dimer filling in the fourth and last As dimer site in the top layer of the $(2 \times 4)$ unit cell. Since this binding energy is much lower than the binding energies for an As ad-dimer in other sites which we have found to be ultimately unstable at standard growth temperatures, ${ }^{11}$ we conclude that the filling in of the fourth dimer site on the local $\beta$ surface should not play a major role as an intermediate configuration during standard growth of the $\beta 2$ surface. Moreover, we find it to be more favorable for an $\mathrm{As}_{2}$ molecule adsorbing on a local $\beta$ structure to attach itself onto the As top layer, with its axis oriented perpendicularly to the existing As dimers. We expect that complete filling of the trench does not occur until nucleation of the new mountains of the next layer up changes the structure so that it is no longer locally the $\beta$ structure.

We note that it may not be easy to distinguish experimentally between a three-dimer $\beta$ reconstruction and a fourdimer completely filled trench: In STM images, the filled dangling bond orbitals of the As dimers in the top layer extend out far enough that the bright stripes corresponding to the two As dimers of the mountain appear considerably wider than the dark stripes corresponding to the two missing As dimers of the trench in the $\beta 2$ regions. It seems quite a)

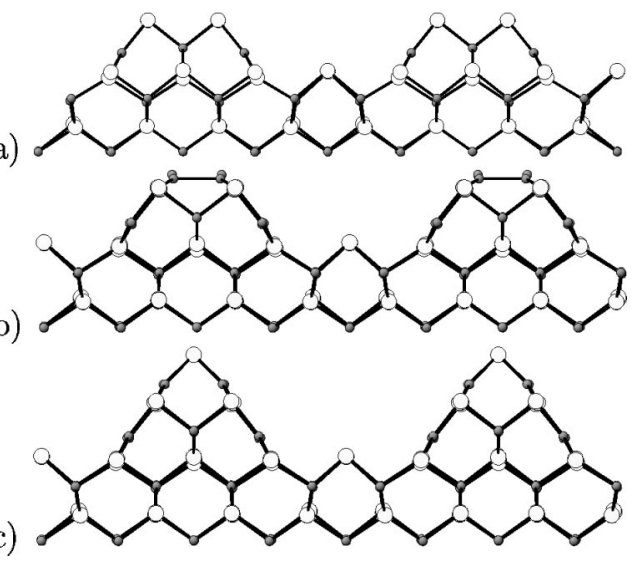

FIG. 5. Growth scenario with nucleation of a new layer, starting from the clean $\beta 2$ surface (a), nucleation of the four-atom Ga cluster $A_{4} A_{2} A_{4}^{\prime \prime} A_{2}^{\prime \prime}$ (b), and $\mathrm{As}_{2}$ adsorption on the Ga cluster (c).

plausible that the three-dimer structure would appear as if it had a completely filled trench in STM pictures, since the filled dangling bond orbitals of the three As dimers in the top layer could extend far enough out to mask the fourth empty dimer site.

For nucleation of a new layer, we consider the possibility that the three-Ga atom cluster $A_{4} A_{2} A_{4}^{\prime \prime}$, and the four-Ga atom cluster $A_{4} A_{2} A_{4}^{\prime \prime} A_{2}^{\prime \prime}$ [Fig. 5(b)] could gain in stability by getting "capped" with $\mathrm{As}_{2}$ [Fig. 5(c)]. Similar to adsorption in the trench, we find that an $\mathrm{As}_{2}$ molecule with only three backbonds is rather weakly bound, by $1.7 \mathrm{eV}$, and thus can only play the role of an intermediary species in growth. However, for the four-Ga atom cluster in the top layer, we find that $\mathrm{As}_{2}$ binds even more strongly there than on a cluster of $\mathrm{Ga}$ adatoms in the trench. The binding energy for an As ad dimer on this four-atom cluster on the mountain is $2.7 \mathrm{eV}$. This indicates that under the usual growth conditions, any such four-atom cluster which forms is likely to be rapidly "capped" by an As ad-dimer, becoming a very stable nucleus for the mountains of the next layer up. Since the Ga adatoms which fall on any local area are likely to migrate to the trench sites before such a four-atom cluster on the mountain has a chance to form, until the nearby Ga sites in the trench are completely filled, we expect that growth will generally proceed by a partial filling of the trenches, and formation of local regions of the $\beta$ structure, followed by nucleation of the mountains of the next layer up in regions adjacent to locally filled trenches. This suggested growth sequence is shown in Fig. 6. Since nucleation of the new mountain is expected to proceed before the fourth As dimer adsorbs, completely filling the trench, we see that the new mountain must nucleate above the center or above the sloping sides of the mountains of the original layer-not above the center of the original trench. This may explain why the new mountains are observed to grow above the old mountains in STM pictures, ${ }^{3}$ instead of above the trenches.

To make quantitative statements about the importance of arsenic adsorption for stabilizing structures during growth, we need to take into account the actual conditions in the growth chamber. In the next section we discuss how this can be achieved in a growth model. 

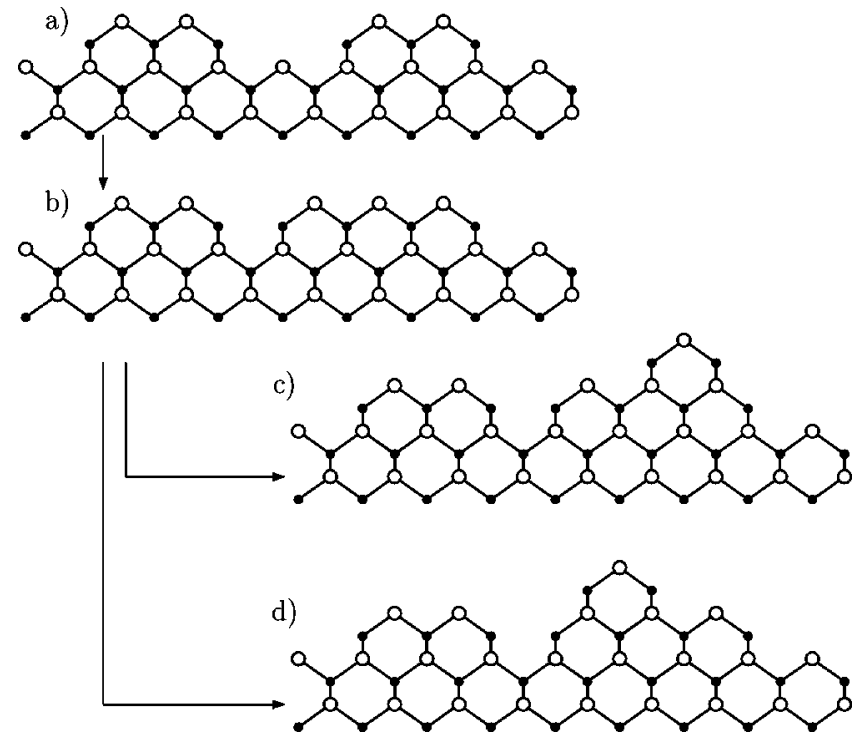

FIG. 6. Growth scenario proposed as a summary of the calculated energetics (schematic): the local $\beta$ reconstruction (b) acts as a precursor for nucleation of the new layer (c) or (d).

\section{IMPLICATIONS FOR A GROWTH MODEL}

In empirical growth models for GaAs, often only the Ga atoms are treated explicitly. ${ }^{14}$ This approach is justified if the concentration of As atoms on the growing surface is completely determined by thermodynamic equilibrium. In MBE growth the flux of incoming As molecules is much higher (typically by a factor of 5) than the number of As atoms per unit area and time that are eventually incorporated into the crystal (which is equal to Ga flux). Therefore the net fluxes of adsorbing and desorbing $\mathrm{As}_{2}$ molecules almost balance, and the concentration of As atoms can be considered as being close to equilibrium. ${ }^{15}$ In particular, this may be true if the residence time of the arsenic molecules is enhanced by a physisorbed precursor state. ${ }^{13}$ Under the equilibrium assumption, the chemical potential of arsenic, $\mu_{\mathrm{As}}(p, T)$, is the same both inside the crystal and in the gas phase, and is determined by the sample temperature $T$ and arsenic partial pressure $p$ in the growth chamber. To be specific, we consider growth from $\mathrm{As}_{2}$. The gas phase chemical potential can be written explicitly:

$$
\begin{aligned}
\mu_{\mathrm{As}}^{\mathrm{As}_{2}}(p, T)= & -E_{\text {coh }}^{\mathrm{As}_{2}}-T S_{\text {vib }}(T)-T S_{\text {rot }}(T) \\
& -T\left[S_{\text {trans }}(p, T)-\frac{5}{2} k\right] .
\end{aligned}
$$

For the cohesive energy of $\mathrm{As}_{2}$, we employ our calculated result of $2.1 \mathrm{eV} /$ atom, which is in fair agreement with the experimental value of $1.96 \mathrm{eV} /$ atom. ${ }^{16}$ The entropies of vibration, rotation, and translation are calculated using the standard textbook expressions, ${ }^{17}$ with the experimental data on spectral properties as input data.

Due to the strong binding of $\mathrm{Ga}$ atoms in $A_{3}$ sites, the evaporation of $\mathrm{Ga}$ adatoms is negligible compared to the incoming flux for temperatures below $1000 \mathrm{~K}$. In contrast to Ref. 15, we therefore argue that the local concentration of $\mathrm{Ga}$ adatoms cannot be described by an equilibrium assumption, but should be treated by a kinetic model. The incoming flux of $\mathrm{Ga}$ atoms drives the system out of equilibrium. Only deep

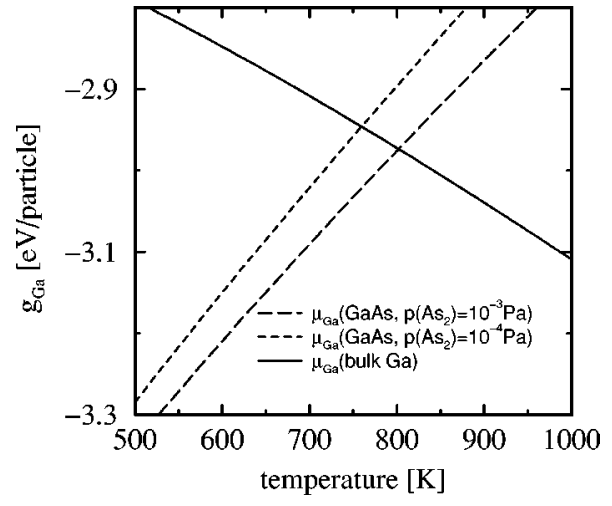

FIG. 7. Free enthalpy per Ga atom in bulk GaAs in thermodynamic equilibrium with $\mathrm{As}_{2}$ vapor at two pressures, compared to the free enthalpy per $\mathrm{Ga}$ atom in elemental $\mathrm{Ga}$.

in the GaAs bulk, a chemical potential for $\mathrm{Ga}$ atoms, $\mu_{\mathrm{Ga}}^{\mathrm{GaAs} b u l k}$, can be defined by the law of mass action for formation of GaAs from the elements,

$$
\mu_{\mathrm{Ga}}^{\mathrm{GaAs} b u l k}(p, T)=\mu_{\mathrm{GaAs}}(T)-\mu_{\mathrm{As}}(p, T) .
$$

Substituting $\mu_{\mathrm{As}}(p, T)$ from Eq. (2), this equation determines the free enthalpy per particle a $\mathrm{Ga}$ atom finally reaches after incorporation. For various adsorption configurations, labeled $i$, the free enthalpy per particle $g_{\mathrm{Ga}}^{(i)}$ is still well-defined even under nonequilibrium conditions, but no chemical potential can be specified. Differences between $g_{\mathrm{Ga}}$ for $\mathrm{Ga}$ atoms in the beam, at the surface and in the bulk act as driving forces for incorporation and growth.

In Fig. 7 , we compare $\mu_{\mathrm{Ga}}^{\mathrm{GaAs} b u l k}$ to $\mu_{\mathrm{Ga}}$ in the bulk of elemental gallium. By virtue of Eq. (3), $\mu_{\mathrm{Ga}}^{\mathrm{Ga} a}$ bulk becomes a function of temperature and $\mathrm{As}_{2}$ background pressure. For the condensed phases of both GaAs and elemental Ga, we have assumed that the pressure dependence of $\mu$ may be neglected. The temperature dependence of $\mu$ for the condensed phase is calculated from a Debye model for the lattice vibrations,

$$
\begin{gathered}
\mu_{\mathrm{Ga}}(T)=-E_{\mathrm{coh}}^{\mathrm{Ga}}+\int_{0}^{T} d T^{\prime} c_{V}^{\mathrm{Ga}}\left(T^{\prime}\right)\left(T^{\prime}-T\right) / T^{\prime} \\
\mu_{\mathrm{GaAs}}(T)=-2 E_{\mathrm{coh}}^{\mathrm{GaAs}}+2 \int_{0}^{T} d T^{\prime} c_{V}^{\mathrm{GaAs}}\left(T^{\prime}\right)\left(T^{\prime}-T\right) / T^{\prime}, \\
c_{V}(T)=9 k_{B}\left(T / T_{D}\right)^{3} \int_{0}^{T_{D} / T} d x \frac{x^{4} e^{x}}{\left(e^{x}-1\right)^{2}} .
\end{gathered}
$$

Here $E_{\text {coh }}$ is the calculated cohesive energy per atom, $3.1 \mathrm{eV}$ for $\mathrm{GaAs}$ and $2.6 \mathrm{eV}$ for $\mathrm{Ga}$, and $c_{V}(T)$ is the specific heat per atom of GaAs and of the bulk phase of $\mathrm{Ga}$, respectively. The Debye temperature $T_{D}$ was taken to be $344 \mathrm{~K}$ for $\mathrm{GaAs},{ }^{18}$ and $240 \mathrm{~K}$ for $\mathrm{Ga} .{ }^{19}$

Figure 7 shows that a background pressure of $\mathrm{As}_{2}$ in the range of $10^{-4} \mathrm{~Pa}$ to $10^{-3} \mathrm{~Pa}$ is required to stabilize the $\mathrm{GaAs}$ crystal under typical growth temperatures, between 700 and $800 \mathrm{~K}$. For $10^{-3} \mathrm{~Pa} \mathrm{As}_{2}$ pressure, the GaAs surface will become unstable against formation of gallium droplets at temperatures above $\sim 800 \mathrm{~K}$. 
a)

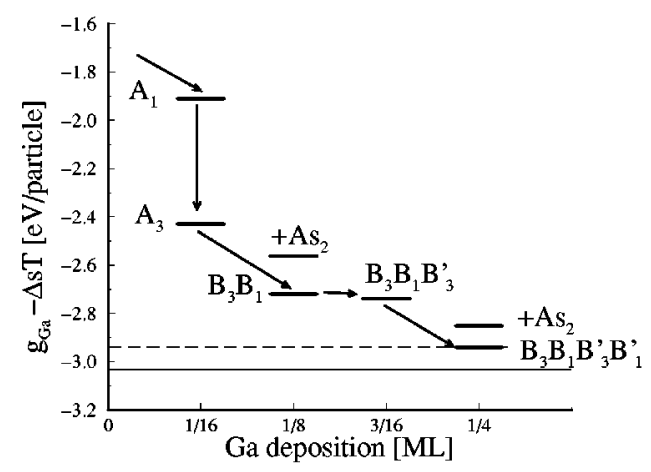

b)

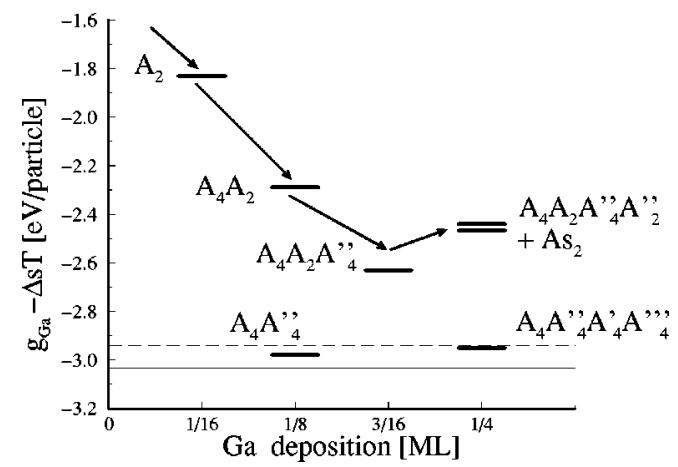

FIG. 8. Free enthalpy per $\mathrm{Ga}$ atom at $T=750 \mathrm{~K}$ and $p\left(\mathrm{As}_{2}\right)$ $=10^{-3} \mathrm{~Pa}$ for two kinetic pathways marked by the arrows, (a) for filling of the trenches (see also Fig. 4), and (b) for nucleation of a new layer (see also Fig. 5). The full and dashed lines indicate the chemical potential of a $\mathrm{Ga}$ atom in the GaAs bulk and in elemental $\mathrm{Ga}$, respectively.

The free enthalpy per atom introduced above makes it possible to compare the energetics of structures on the kinetic pathway of growth that contain different amounts of gallium and arsenic. From our ab initio calculations, we obtain the relative energies $\Delta e^{(i)}$ of various adsorbate structures on $\operatorname{GaAs}(001)$ at $p=0$ and $T=0$ with respect to a reservoir of elemental Ga. The transfer of a $\mathrm{Ga}$ atom from the reservoir into the $A_{3}$ site on the surface is endothermic by $0.55 \mathrm{eV}$. The values of $\Delta e^{(i)}$ can be read from Table I using the relation $\Delta e^{(i)}=0.55 \mathrm{eV}+\Delta E^{(i)} / N_{\mathrm{Ga}}$, where $N_{\mathrm{Ga}}$ is the number of deposited $\mathrm{Ga}$ atoms. Formally, each deposited As atom goes along with the transfer of a $\mathrm{Ga}$ atom from the $\mathrm{Ga}$ bulk to the GaAs bulk reservoir, leading to a free enthalpy change equal to $\mu_{\mathrm{Ga}}^{\mathrm{GaAs} b u l k}(p, T)-\mu_{\mathrm{Ga}}(T)$. Hence we can directly compute the quantity

$$
\begin{aligned}
g_{\mathrm{Ga}}^{(i)}+\Delta s^{(i)} T= & \mu_{\mathrm{Ga}}(T)+\Delta e^{(i)}+\left(\mu_{\mathrm{Ga}}^{\mathrm{GaAs} b u l k}(p, T)\right. \\
& \left.-\mu_{\mathrm{Ga}}(T)\right) N_{\mathrm{As}} / N_{\mathrm{Ga}},
\end{aligned}
$$

with $N_{\text {As }}$ the number of deposited As atoms, and $\Delta s^{(i)}$ being the difference in entropy of a $\mathrm{Ga}$ atom in adsorption site $i$ and in the Ga bulk. This difference contains contributions from vibrational entropy as well as from configurational entropy, which in turn depends on the concentration of deposited $\mathrm{Ga}$ adatoms on the surface.
A gallium atom stemming from the beam source typically runs through several intermediate configurations on the surface before it becomes part of the GaAs solid. During this process, its free enthalpy $g_{\mathrm{Ga}}$ decreases gradually. Figure 8 illustrates the approach of $g_{\mathrm{Ga}}$ towards its equilibrium value in the GaAs bulk for the two growth scenarios discussed above. The quantity $g_{\mathrm{Ga}}^{(i)}+\Delta s^{(i)} T$ is shown for deposition of an increasing number of $\mathrm{Ga}$ atoms. Whenever competing structures with or without As "capping" are expected, we compare the free enthalpy of both. The values shown in the figure are calculated for $T=750 \mathrm{~K}$ and $p=10^{-3} \mathrm{~Pa}$. Under these conditions, incorporation of two Ga atoms in the local $\beta$ structure (labeled $B_{3} B_{1}+\mathrm{As}_{2}$ ) has a slightly higher free enthalpy than the local $\alpha$ structure $\left(B_{3} B_{1}\right)$. Thus, the majority of the two-Ga clusters formed in the trenches will be uncovered after their formation. For longer chains of Ga atoms filling up the trench, the situation is similar: Larger patches of $\alpha$ structures (labeled $B_{3} B_{1} B_{3}^{\prime} B_{1}^{\prime}$ ) have a slightly lower free enthalpy than the corresponding As-capped $\beta$ structures. Thus they will be predominantly uncovered under the considered conditions. The situation changes somewhat at temperatures below $700 \mathrm{~K}$ or higher As pressures, when As-covered $\beta$ structures become equally probable. For the structures nucleated on the top layer, the calculations show that the $\mathrm{As}_{2}$ molecule binds strongly to the four-atom $\mathrm{Ga}$ cluster. Therefore the As-covered structure is preferred under a wide range of growth conditions where the surface As concentration is in equilibrium with gas phase $\mathrm{As}_{2}$. However, as can be seen from Fig. 8, individual Ga dimers $\left(A_{4} A_{4}^{\prime \prime}\right)$ or Ga dimers above pairs of As dimers, separated by a gap $\left(A_{4} A_{4}^{\prime \prime} A_{4}^{\prime} A_{4}^{\prime \prime \prime}\right)$, are still the most stable species in absolute terms.

Since the kinetics of Ga incorporation is driven by differences in $g_{\mathrm{Ga}}$, a kinetic model of growth that includes only the Ga species should describe the stability of different configurations in terms of the quantities $g_{\mathrm{Ga}}^{(i)}$. The effective parameters of such a model depend on temperature and arsenic background pressure. Equation (7) makes it possible to determine them from first principles, apart from the yet undetermined entropic contribution $\Delta s T$. In a kinetic model, all microscopic processes are represented by their respective rates. In addition to knowledge of the (meta-)stable configurations occurring during growth, a microscopic determination of the rates also requires knowledge about the transition states. Within classical rate theory, the rate for a particular transition is given by $\Gamma=\exp \left(\left(g^{(i)}-g^{T S}\right) / k T\right)$, with the free enthalpy at the transition state, $g^{T S}$. It is frequently represented by an activation energy and a prefactor, $\Gamma$ $=\Gamma_{0} \exp \left(-E_{A} / k T\right)$. When rates are determined from calculated free enthalpies, the contributions to $\Delta s T$ from configurational entropy should be omitted, since they are described implicitly by the number of microscopic processes possible in a given situation. The vibrational entropies enter the rates only through the prefactor, but do not appear in the activation energy. Without a detailed calculation of vibrational properties, one has to rely on the assumption that vibrational contributions to the entropy are similar in different configurations. Thus they tend to cancel out in $\Delta s$ and will not qualitatively change the picture. This is equivalent to the 
assumption of a common prefactor for all kinetic processes, as is frequently made in kinetics simulations.

\section{CONCLUSION}

In conclusion, we have investigated the energetics of two kinetic pathways for homoepitaxy on $\operatorname{GaAs}(001)$ by means of density functional theory calculations. We find that two gallium adatoms on the GaAs(001) surface interact sufficiently strongly to form stable nuclei. One type of nuclei consists of $\mathrm{Ga}$ atoms in adjacent threefold coordinated sites in the trenches, while another type consists of $\mathrm{Ga}$ dimers forming on the top layer of arsenic. Since single Ga atoms in the top layer are energetically unfavorable and constitute an excited state, the formation of Ga dimers in the top layer is suppressed at low coverages, and growth will be dominated by the nucleation of $\mathrm{Ga}$ atom pairs in the trenches. Filling of the trenches will proceed by further attachment of $\mathrm{Ga}$ adatoms to these nuclei. From our calculations, we expect that islands in the new layer will preferentially form in those regions of the surface where locally the $\beta$ reconstruction has formed, i.e., where the trenches have been partially filled.

Upon further deposition of material, the islands start to grow. Quenched STM images have revealed small islands which do not yet show the $(2 \times 4)$ pattern. ${ }^{3}$ After passing through this metastable intermediate state, eventually the islands will restructure and display the trenches characteristic for the $\beta 2$ reconstruction.
${ }^{1}$ H. Farrel, J.P. Harbison, and L.D. Peterson, J. Vac. Sci. Technol. B 5, 1482 (1987).

${ }^{2}$ A.R. Avery, H.T. Dobbs, D.M. Holmes, B.A. Joyce, and D.D. Vvedensky, Phys. Rev. Lett. 79, 3938 (1997).

${ }^{3}$ M. Itoh et al., Phys. Rev. Lett. 81, 633 (1998).

${ }^{4}$ J.P. Perdew, K. Burke, and M. Ernzerhof, Phys. Rev. Lett. 77, 3865 (1996).

${ }^{5}$ D.R. Hamann, Phys. Rev. B 40, 2980 (1989).

${ }^{6}$ L. Kleinman and D.M. Bylander, Phys. Rev. Lett. 48, 1425 (1982).

${ }^{7}$ M. Fuchs and M. Scheffler, Comput. Phys. Commun. 116, 1 (1999).

${ }^{8}$ X. Gonze, R. Stumpf, and M. Scheffler, Phys. Rev. B 44, 8503 (1991).

${ }^{9}$ A. Kley, P. Ruggerone, and M. Scheffler, Phys. Rev. Lett. 79, 5278 (1997).

${ }^{10}$ Estimate based on the hopping rate along the [110] direction at 800 K [Eq. (5) in Ref. 9].

${ }^{11}$ C.G. Morgan, P. Kratzer, and M. Scheffler, Phys. Rev. Lett. (to be published).

${ }^{12}$ K. Shiraishi and T. Ito, Surf. Sci. 357-358, 451 (1996); Phys. Rev. B 57, 6301 (1998).

${ }^{13}$ C.T. Foxon and B.A. Joyce, Surf. Sci. 64, 293 (1977).

${ }^{14}$ D.D. Vvedensky, S. Clarke, K.J. Hugill, A.K. Myers-Beaghton, and M.R. Wilby, Kinetics of Ordering and Growth at Surfaces, edited by M.G. Lagally (Plenum Press, New York, 1988).

${ }^{15}$ J. Tersoff, M.D. Johnson, and B.G. Orr, Phys. Rev. Lett 78, 282 (1997).

${ }^{16}$ Handbook of Chemistry and Physics, edited by R. C. West, 67th ed. (CRC, Boca Raton, 1986).

${ }^{17}$ W. Greiner, L. Neise, and H. Stöcker, Thermodynamik und Statistische Mechanik (Harri Deutsch, Frankfurt am Main, 1987).

${ }^{18}$ D. Bimberg et al., in Numerical Data and Functional Relationships in Science and Technology, edited by K.-H. Hellwege, Landolt-Börnstein, New Series, Group 17, Vol. III, Pts. a, b (Springer-Verlag, Berlin, 1982), p. 642.

${ }^{19}$ N. W. Ashcroft and N. D. Mermin, Solid State Physics (Saunders College, Philadelphia, 1976). 